\title{
Clinical characteristics and prognosis of pediatric patients with B cell acute lymphoblastic leukemia relapse
}

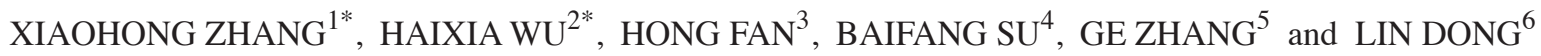 \\ ${ }^{1}$ Department of Pediatrics, Jining First People's Hospital, Jining, Shandong 272000; ${ }^{2}$ Department of Pediatrics, \\ Yantaishan Hospital, Yantai, Shandong 264000; Departments of ${ }^{3}$ Nursing, ${ }^{4}$ Science and Education, \\ and ${ }^{5}$ Obstetrics, People's Hospital of Zhangqiu District; ${ }^{6}$ Department of Hematology, \\ Qianfoshan Hospital Affiliated to Shandong University, Jinan, Shandong 250000, P.R. China
}

Received December 14, 2017; Accepted May 16, 2018

DOI: $10.3892 / 01.2018 .8974$

\begin{abstract}
The aim of the present study was to investigate the clinical characteristics and prognosis of pediatric patients with B cell acute lymphoblastic leukemia (B-ALL) relapse. A total of 390 pediatric patients diagnosed as B-ALL and receiving regular chemotherapy in Jining First People's Hospital from August 2010 to May 2016 were selected. The clinical characteristics, therapeutic response and prognosis were compared between the two groups. There were significant differences in the comparisons of age, leukocyte count in the initial diagnosis and glucocorticoid sensitive test between B-cell ALL (B-ALL) relapse group and non-relapse group; the minimal residual disease (MRD) levels of pediatric patients in the two groups at 33 days and 12 weeks were significantly different. The 3-year event-free survival (EFS) rates of pediatric patients with early, medium and late B-ALL relapse were $12.5 \pm 7.8 \%$, $33.1 \pm 9.8 \%$ and $63.6 \pm 6.1 \%$, respectively, and the prognosis of late relapse was significantly better than that of early relapse $(\mathrm{P}<0.001)$. The 3-year EFS rates of pediatric patients with bone marrow relapse in standard risk group, intermediate risk group and high risk group were $29.1 \pm 6.9,31.3 \pm 6.5$ and $28.3 \pm 6.3 \%$, respectively; there were no statistically significant differences $(\mathrm{P}=0.387, \mathrm{P}>0.05)$. Pediatric patients with $\mathrm{B}-\mathrm{ALL}$ relapse are characterized by higher onset age ( $\geq 10$ years old), high leukocyte count and hormone insensitivity. Dynamic monitoring of MRD level in B-ALL pediatric patients can predict the relapse.
\end{abstract}

\section{Introduction}

With the progress in risk stratification, chemotherapy, hematopoietic stem cell transplantation (HSCT) and supportive treatment,

Correspondence to: Dr Lin Dong, Department of Hematology, Qianfoshan Hospital Affiliated to Shandong University, 16766 Jingshi Road, Jinan, Shandong 250000, P.R. China

E-mail: yacongxianyiy@163.com

*Contributed equally

Key words: ALL, event-free survival, relapse, MRD the current overall survival (OS) of acute lymphoblastic leukemia (ALL), as the most common tumor in children, has been more than $80 \%$, but there are still approximately $20 \%$ (1) children with relapse. Most pediatric patients will suffer from relapse again even after the relapse is treated with high-intensity combined chemotherapy and/or allogeneic HSCT, and only approximately one-third of pediatric patients can be cured, so the overall prognosis is not significantly improved (2), and the relapse is still an important risk factor threatening the long-term survival and healing of ALL pediatric patients. The age in the initial diagnosis, leukocyte count, immunophenotyping, cytogenetics, gene characteristics, minimal residual disease (MRD) and early therapeutic response are the main risk factors for assessing the relapse of ALL pediatric patients (3). According to Chinese Children's Leukemia Group (CCLG)-2008-ALL protocol, ALL pediatric patients can be divided into standard risk group, intermediate risk group and high risk group. The relapse rates of pediatric patients in moderate and high risk groups are relatively higher, and the long-term event-free survival (EFS) rates are lower, especially pediatric patients in high risk group, which may be related to the higher age in the initial diagnosis, high leukocyte count, cytogenetic changes and poor early therapeutic response. However, some pediatric patients in standard risk group also suffer from relapse in the medium and late stage, indicating that the current risk stratification cannot predict the prognosis very accurately, and it is needed to find new poor prognosis-related factors as soon as possible and use it in the guidance of clinical stratification and treatment.

Relapse occurs most frequently in bone marrow of ALL pediatric patients. According to the research of Children's Oncology Group (COG), the 5-year EFS rate of intramedullary relapse is approximately $30 \%$ (4), and the 5-year EFS rates of simple intramedullary relapse, simple extramedullary relapse and intra-extramedullary relapse are 24\%, 59\% and $39 \%$, respectively $(5,6)$. The results show that the prognosis of simple extramedullary (central nervous leukemia and testicular leukemia) relapse is the best, and the prognosis of simple bone marrow relapse is inferior to that of intra-extramedullary relapse $(6,7)$. An AAL LOIP2 study performed by COG recently made a conclusion that the relapse time can predict the prognosis; only less than $1 / 3$ pediatric patients with early relapse (relapse within 36 months after the initial diagnosis according to 
COG standard) can survive, while nearly $1 / 2$ pediatric patients with late relapse (relapse at more than 36 months after the initial diagnosis according to COG standard) can survive $(8,9)$. Drug-resistant mutation has occurred at the onset in T-cell ALL (T-ALL) pediatric patients due to rapid cell mutation and low apoptosis rate (10), and there is much residual leukemia cloning; thus early relapse occurs more easily in central nervous system in T-ALL than B-cell ALL (B-ALL). A large number of clinical studies in China and foreign countries have confirmed that the MRD levels at different time points of chemotherapy are closely related to the relapse and long-term EFS, and monitoring the MRD level is conducive to the evaluation of curative effect and adjustment of chemotherapy regimens of $\operatorname{ALL}(11,12)$.

In this study, the clinical characteristics and curative effect of 390 pediatric patients initially diagnosed as B-ALL in Jining First People's Hospital (Jining, China) in the past 6 years were analyzed, and the risk factors related to ALL relapse were searched, so as to lay a foundation for reducing relapse and studying its related gene molecules in the future.

\section{Materials and methods}

Objects of study. A total of 390 pediatric patients admitted and initially diagnosed as B-ALL in Jining First People's Hospital from August 2010 to May 2016 were selected, and they were diagnosed via bone marrow morphology, immunology, cytogenetics and molecular biology (MICM) classification. All pediatric patients were followed up; this study followed the ethical standards set by the Institutional Review Board and was approved by The Ethics Committee of Jining First People's Hospital. Written, informed consent was obtained from all patients or their parents prior to their inclusion within the study.

Risk stratification. The risk of leukemia was stratified according to the age of pediatric patients in the initial diagnosis, peripheral leukocyte count, abnormalities in cytogenetics and molecular biology, whether they were sensitive to prednisone, bone marrow remission status at 33 days and MRD at 33 days; the therapeutic regimen was based on the CCLG-2008 protocol (modified BFM ALL-95 protocol) (13), and the diagnosis and efficacy were based on 'Diagnosis and Treatment Recommendations of Pediatric Acute Leukemia (Revised Draft)'.

Criteria of risk stratification. The risk was stratified according to the age of pediatric patients in the initial diagnosis, peripheral leukocyte count, bone marrow puncture results (immunophenotyping, chromosome and 29 fusion genes) and treatment effects, and ALL pediatric patients could be divided into standard risk (SR) group, intermediate risk (IR) group and high risk (HR) group.

Immunophenotyping. Bone marrow (2-3 ml) was taken from pediatric patients for anticoagulation to separate the karyocytes, followed by immunofluorescence labeling using the living-cell index method (FITC or PE). The antibodies used were all mouse monoclonal antibodies and the dilution was 1/100: CD2 (cat. no. 563820); CD3 (cat. no. 565066); CD5 (cat. no. 564648); CD7 (cat. no. 566119); CD10 (cat. no. 564959); CD19 (cat.no. 564978); CD20 (cat. no. 564918); CD22 (cat.no. 563940); cCD79 (cat. no. 555302); cCD3 (cat. no. 565065); HLA-DR (cat. no. 564516); TdT (cat. no. 565229); MPO (cat. no. 556035) and Smlg (cat. no. 564555). All were purchased from BD Biosciences (San Diego, CA, USA). Flow cytometer (Beckman Coulter, Brea, CA, USA) was used; antigen-expressing cells in juvenile cell group $>20 \%$ indicated the positive.

Chromosome and fusion gene detection. The chromosomes were taken from the bone marrow of pediatric patients, and the chromosome samples were prepared using the direct method or $24 \mathrm{~h}$ short-term culture. The karyotype was analyzed using the conventional R-banding technique. The chromosome abnormality was explained based on the International System for Human Cytogenetic Nomenclature (ISCN1985) (14). Bone marrow (2-3 $\mathrm{ml}$ ) with heparin anticoagulation was taken in the initial diagnosis, and the mononuclear cells were extracted using lymphocyte analytic liquid (Ficoll). Twenty-nine fusion genes [including ETV6/RUNXI (TEL/AML1), MLL rearrangement, HOX11, E2A/PBX1, and BCR/ABL] were detected via nested reverse transcription polymerase chain reaction (RT-PCR).

MRD screening and monitoring. Bone marrow $(2-3 \mathrm{ml})$ with heparin anticoagulation was taken from pediatric patients in the initial diagnosis before chemotherapy, and the karyocytes were separated routinely and counted. Then the karyocytes were stained using CD45-FITC grading, CD10-PE, CD34-Percp and CD19-APC monoclonal antibodies; the antibody combination was effective if the leukemia cells and normal bone marrow cells could be distinguished. At 33 days and 12 weeks after induction chemotherapy, the bone marrow samples were taken from pediatric patients, and the mononuclear cells were separated using Ficoll, followed by four-color fluorescence labeling detection (CD58, CD66c, CD38, CD123 and TdT were added based on CD34/CD19/CD10); the original data in the initial diagnosis and screening were called up; in the two-parameter diagram, if there were cells in the original area of leukemia cells, it indicated the minimal residual leukemia; the proportion of these residual cells in total bone marrow mononuclear cells was the result of monitoring MRD.

Therapeutic regimen. Among the newly-diagnosed pediatric patients selected, except those with mature-type B-ALL $(n=12)$ who were treated as non-Hodgkin's lymphoma, all the other pediatric patients were treated according to CCLG-2008-ALL protocol and chemotherapy regimens with different intensities according to different risk degrees as follows: i) remission induction: pretreatment with prednisone for 7 days, VDLD (vincristine, daunorubicin, L-asparaginase or pegaspargase, dexamethasone) regimen in induction; ii) early intensive treatment: CAM (cyclophosphamide, cytarabine, 6-mercaptopurine or thioguanine) program (SR group $\mathrm{x}$ one round; IR and HR groups $x$ two rounds); iii) consolidation therapy: SR and IR groups: HD-methotrexate (MTX) + 6-MP program ( $2.0 \mathrm{~g} / \mathrm{m}^{2}$ MTX in SR group, $5.0 \mathrm{~g} / \mathrm{m}^{2}$ MTX in IR group); HR group: HR-1,HR-2 and HR-3 (a total of two rounds); iv) delayed enhancement: the same VDLD and CAM programs as above [two rounds of delayed enhancement for IR group (one round of maintenance chemotherapy between the two rounds of delayed enhancement)]; v) maintenance treatment: 6-MP + MTX. The single or triple chemotherapy drugs were regularly injected intrathecally to prevent the central nervous system leukemia. 
Table I. Comparison of clinical features of relapse and non-relapse of B-ALL.

\begin{tabular}{|c|c|c|c|}
\hline Clinical features & Relapse group & Non-relapse group & P-value \\
\hline Number (n) & 80 & 310 & \\
\hline Sex & & & 0.421 \\
\hline Male & $54(67.5 \%)$ & $173(55.8 \%)$ & \\
\hline Female & $26(32.5 \%)$ & $137(44.2 \%)$ & \\
\hline Age (years) & & & 0.031 \\
\hline$<1$ & 0 & $2(0.6 \%)$ & \\
\hline $1-10$ & $68(85 \%)$ & $280(90.3 \%)$ & \\
\hline$\geq 10$ & $12(15 \%)$ & $28(9.1 \%)$ & \\
\hline WBC in the initial diagnosis & & & 0.032 \\
\hline$<50 \times 10^{9} / 1$ & $51(63.8 \%)$ & $264(85.2 \%)$ & \\
\hline$\geq 50 \times 10^{9} / 1$ & $29(36.2 \%)$ & $46(14.8 \%)$ & \\
\hline Response of prednisone pretreatment & & & 0.01 \\
\hline Sensitive & $60(75 \%)$ & $287(92.6 \%)$ & \\
\hline Non-sensitive & $20(25 \%)$ & $23(7.4 \%)$ & \\
\hline MRD (33 days) & & & 0.015 \\
\hline$<10^{-2}$ & $65(81.2 \%)$ & $290(93.5 \%)$ & \\
\hline$\geq 10^{-2}$ & $15(18.8 \%)$ & $20(6.5 \%)$ & \\
\hline MRD (12 weeks) & & & 0.001 \\
\hline$<10^{-3}$ & $56(70 \%)$ & $282(91.0 \%)$ & \\
\hline$\geq 10^{-3}$ & $24(30 \%)$ & $28(9 \%)$ & \\
\hline Abnormal chromosomes & $36(45 \%)$ & $162(52.2 \%)$ & 0.890 \\
\hline Fusion genes & $21(26.3 \%)$ & $108(34.8 \%)$ & 0.045 \\
\hline Risk stratification & & & 0.013 \\
\hline SR & $21(26.3 \%)$ & $98(31.6 \%)$ & \\
\hline IR & $26(32.5 \%)$ & $121(39.0 \%)$ & \\
\hline HR & $33(41.2 \%)$ & $91(29.4 \%)$ & \\
\hline After the first course of treatment & & & 0.654 \\
\hline Remission & $69(86.3 \%)$ & $273(88.1 \%)$ & \\
\hline Non-remission & $11(13.7 \%)$ & $37(11.9 \%)$ & \\
\hline
\end{tabular}

Therapeutic regimen of relapse (15). Re-induction therapy: VMDP (vincristine, mitoxantrone, dexamethasone, pegaspargase). Intensive consolidation therapy: FLAG (fludarabine, HD-cytarabine, granulocyte-stimulating factor) or CAM + 6-MP. Transplantation or continuous treatment [delayed enhancement (vincristine, dexamethasone, methotrexate, 6-MP, cyclophosphamide, cytarabine, etoposide) + maintenance chemotherapy (vincristine, dexamethasone, methotrexate, 6-MP)]. Extramedullary relapse: local radiotherapy after chemotherapy. CNSL: head radiotherapy $1800 \mathrm{cGy}, 150 \mathrm{cGy} / \mathrm{day}, 12$ days; TL: testicular local radiotherapy 2400 cGy, 200 cGy/day, 12 days.

Efficacy evaluation. Complete remission (CR): the primitive and juvenile lymphocytes $<5 \%$ on the bone marrow smear after induction chemotherapy. Relapse: including simple intramedullary relapse, extramedullary relapse (central nervous system or testicular relapse) and intra-extramedullary relapse.
Statistical analysis. Patients were followed up until December 31, 2015, the median follow-up time was 22.7 months (2.8-62.4 months), and there were a total of 80 cases of relapse. EFS: the time from relapse to relapse again or death. Statistical Product and Service Solutions (SPSS, Chicago, IL, USA) 17.0 Software Package was used for the comparison of 3-year EFS rate of B-ALL pediatric patients in intramedullary relapse group at different time points; Kaplan-Meier survival curve analysis method was used for the comparison of 3-year EFS rate in different risk degrees; bilateral log-rank test was used for the survival situations in different groups. The independentsamples t-test was used for the clinical characteristics between B-ALL relapse group and non-relapse group, and Chi-square test was used for the comparison of constituent ratio of clinical characteristics between the two groups. $\mathrm{P}<0.05$ was considered to indicate a statistically significant difference. 
Table II. Comparisons of chromosomes between relapse group and non-relapse group with B-ALL.

\begin{tabular}{lccc} 
Items & Normal chromosome & $\begin{array}{c}\text { Abnormal chromosome } \\
\text { quality }\end{array}$ & $\begin{array}{c}\text { Abnormal chromosome } \\
\text { counts }\end{array}$ \\
\hline Relapse group & $54.9 \%(44 / 80)$ & $21.3 \%(17 / 80)$ & $23.8 \%(19 / 80)$ \\
Non-relapse group & $52.9 \%(164 / 310)$ & $21.9 \%(68 / 310)$ & $25.2 \%(78 / 310)$ \\
P-value & 0.483 & 0.873 & 0.678 \\
\hline
\end{tabular}

Table III. Comparisons of fusion genes between relapse group and non-relapse group of B-ALL.

\begin{tabular}{llr}
\hline Items & \multicolumn{1}{c}{ Fusion genes } & Normal genes \\
\hline Relapse group & $83.8 \%(67 / 80)$ & $16.2 \%(13 / 80)$ \\
Non-relapse group & $61.0 \%(189 / 310)$ & $39 \%(121 / 310)$ \\
P-value & \multicolumn{2}{c}{0.032} \\
\hline
\end{tabular}

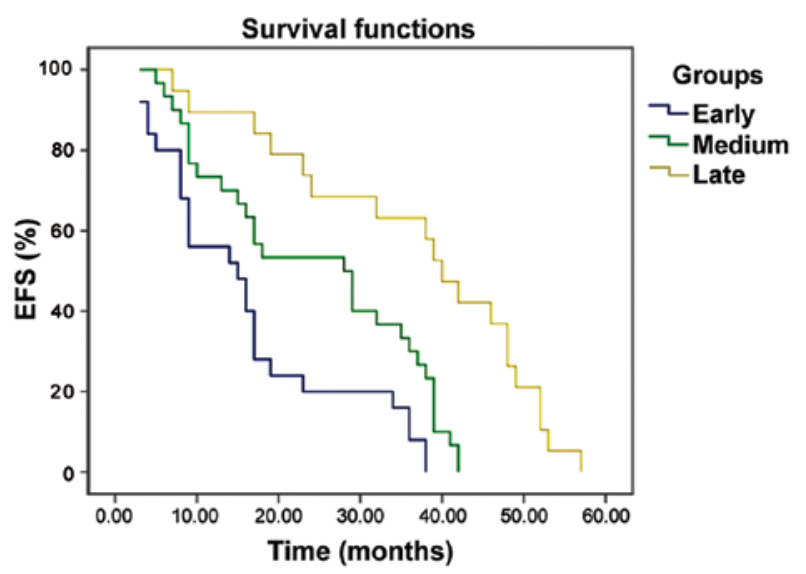

Figure 1. The EFS curve of B-ALL pediatric patients with early, medium and late bone marrow relapse. Kaplan-Meier survival curve analysis method was used for the comparison of 3-year EFS rate in different groups; bilateral log-rank test was used for the survival situations in different groups.

\section{Results}

General data. The general data of subjects in the two groups are shown in Table I. There was a significant difference in the proportion of B-ALL pediatric patients with different risk stratification in relapse group and non-relapse group; the pairwise comparison among SR group, IR group and HR group showed that the difference in the proportion of relapse in B-ALL pediatric patients was not statistically significant between SR group and IR group, but the difference was statistically significant compared with that in HR group $(\mathrm{P}=0.013)$. The comparison of pediatric patients in B-ALL relapse group and non-relapse group revealed that there was no statistically significant difference in the sex composition between the two groups $(\mathrm{P}=0.421, \mathrm{P}>0.05)$, but there were statistically significant differences in the age and ratio of white blood cell $(\mathrm{WBC}) \geq 50 \times 10^{9} / 1$ in the initial diagnosis $(\mathrm{P}=0.031, \mathrm{P}=0.032$, $\mathrm{P}<0.05)$.

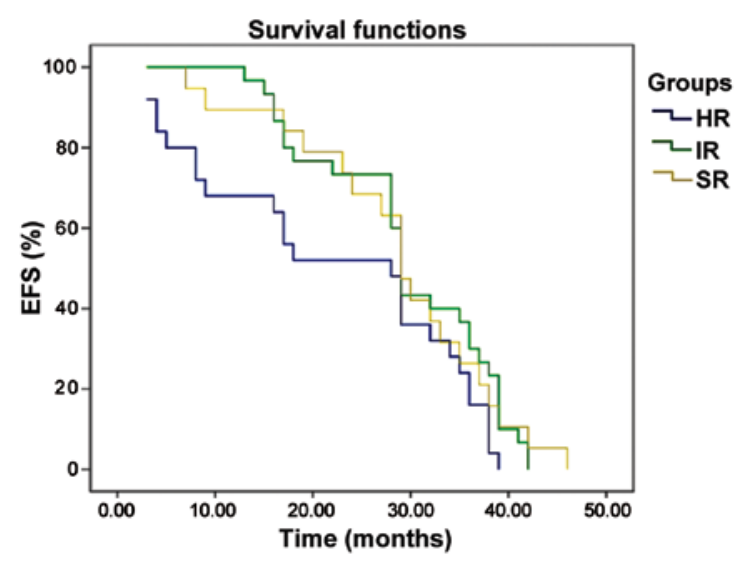

Figure 2. The EFS curve of B-ALL pediatric patients with bone marrow relapse in SR group, IR group and HR group. Kaplan-Meier survival curve analysis method was used for the comparison of 3-year EFS rate in different risk degrees; bilateral log-rank test was used for the survival situations in different groups.

Cytogenetics and molecular biology results. The cytogenetics and molecular biology results of pediatric patients with relapse and non-relapse in B-ALL group are shown in Tables II and III. For chromosome analysis, we did not detect significant difference between two groups while for gene analysis, fusion gene rate in the relapse group was significantly higher than that in the non-relapse group.

Analysis of MRD monitoring at different time points. MRD of pediatric patients with B-ALL relapse and non-relapse was monitored at 33 days and 12 weeks after chemotherapy, and they were divided according to different MRD levels for analysis and comparison (Tables IV and V). At day 33, there were more children relapsed in the $M R D \geq 10^{-2}$ group than that in $\mathrm{MRD}<10^{-2}$ group $(\mathrm{P}<0.05)$. At week 12 , there were more children relapsed in the $\mathrm{MRD} \geq 10^{-3}$ group than that in MRD $<10^{-3}$ group $(\mathrm{P}<0.05)$. The relapse rate in the $<10^{-4}$ group was lower than non-relapse rate $(\mathrm{P}<0.05)$. Moreover, the ratio in $10^{-4}-10^{-3}, 10^{-3}-10^{-2}$ and $\geq 10^{-2}$ in relapse group was higher than that in non-relapse group. As a result, MRD $<10^{-4}$ could be thought as a cut-off point of low relapse rate.

Relapse site and time. The relapse site and time of pediatric patients in the relapse group and their relationship with risk typing are shown in Tables VI and VII. As defined by BerlinFrankfurt-Munster-ALL Group, early relapse: the time from initial diagnosis to relapse was $\leq 18$ months; medium relapse: the time from initial diagnosis to relapse was $>18$ months and $\mathrm{CR}<30$ months; late relapse: $\mathrm{CR}>30$ months. 
Table IV. Comparisons of MRD at 33 days between relapse and non-relapse group with B-ALL.

\begin{tabular}{|c|c|c|c|c|c|c|}
\hline \multirow[b]{2}{*}{ Items } & \multirow[b]{2}{*}{$\mathrm{CR}$} & \multirow[b]{2}{*}{ Non-CR } & \multicolumn{4}{|c|}{ MRD at 33 days } \\
\hline & & & $<10^{-4}$ & $10^{-4}-10^{-3}$ & $10^{-3}-10^{-2}$ & $\geq 10^{-2}$ \\
\hline Relapse group & 70 & 10 & $32(40 \%)$ & $24(30.0 \%)$ & $12(15 \%)$ & $12(15 \%)$ \\
\hline Non-relapse group & 243 & 67 & $173(55.8 \%)$ & $88(28.4 \%)$ & $45(14.5 \%)$ & $4(1.3 \%)$ \\
\hline P-value & \multicolumn{2}{|c|}{$P>0.05$} & 0.043 & 0.122 & 0.435 & 0.001 \\
\hline
\end{tabular}

Table V. Comparisons of MRD at 12 weeks between relapse and non-relapse group with B-ALL.

\begin{tabular}{|c|c|c|c|c|c|c|}
\hline \multirow[b]{2}{*}{ Items } & \multirow[b]{2}{*}{$\mathrm{CR}$} & \multirow[b]{2}{*}{ Non-CR } & \multicolumn{4}{|c|}{ MRD at 12 weeks } \\
\hline & & & $<10^{-4}$ & $10^{-4}-10^{-3}$ & $10^{-3}-10^{-2}$ & $\geq 10^{-2}$ \\
\hline Relapse group & 68 & 12 & $24(30 \%)$ & $29(36.3 \%)$ & $18(22.5 \%)$ & $9(11.2 \%)$ \\
\hline Non-relapse group & 233 & 77 & $162(52.3 \%)$ & $102(32.9 \%)$ & $25(8.1 \%)$ & $21(6.7 \%)$ \\
\hline P-value & \multicolumn{2}{|c|}{$P>0.05$} & 0.032 & 0.546 & 0.001 & 0.002 \\
\hline
\end{tabular}

Table VI. The relapse site of 80 B-ALL cases.

\begin{tabular}{lccc}
\hline & $\begin{array}{c}\text { Central nervous } \\
\text { system }\end{array}$ & Testis & $\begin{array}{c}\text { Combining } \\
\text { relapse }\end{array}$ \\
\hline $88.8 \%(71 / 80)$ & $5 \%(4 / 80)$ & $5 \%(4 / 80)$ & $3.8 \%(3 / 80)$
\end{tabular}

Table VII. Relapse stages of different risk stratifications of B-ALL.

\begin{tabular}{lcccc}
\hline & \multicolumn{4}{c}{ Risk stratification } \\
\cline { 2 - 5 } $\begin{array}{l}\text { Stage of } \\
\text { relapse }\end{array}$ & $\begin{array}{c}\text { Standard } \\
\text { risk }\end{array}$ & $\begin{array}{c}\text { Intermediate } \\
\text { risk }\end{array}$ & $\begin{array}{c}\text { High } \\
\text { risk }\end{array}$ & $\begin{array}{c}\text { Total } \\
\text { number }\end{array}$ \\
\hline Early stage & 2 & 12 & 20 & 34 \\
Medium stage & 8 & 11 & 19 & 38 \\
Late stage & 5 & 2 & 1 & 8 \\
\hline
\end{tabular}

Long-term survival analysis. The EFS curve of B-ALL pediatric patients with early, medium and late bone marrow relapse, the EFS curve of B-ALL pediatric patients with bone marrow relapse in SR group, IR group and HR group are shown in Figs. 1 and 2. The 3-year event-free survival (EFS) rates of pediatric patients with early, medium and late B-ALL relapse were $12.5 \pm 7.8,33.1 \pm 9.8$ and $63.6 \pm 6.1 \%$, respectively, and the prognosis of late relapse was significantly better than that of early relapse $(\mathrm{P}<0.001)$. The 3 -year EFS rates of pediatric patients with bone marrow relapse in standard risk group, intermediate risk group and high risk group were $29.1 \pm 6.9,31.3 \pm 6.5$ and $28.3 \pm 6.3 \%$, respectively; there were no statistically significant differences $(\mathrm{P}=0.387$, $\mathrm{P}>0.05$ ).

\section{Discussion}

ALL is the most common type of AL in children, accounting for approximately $80 \%(15,16)$, whose immunophenotyping is mostly B-lineage expression. In recent years, with the improvement of risk stratification, chemotherapy regimens and HSCT, the cure rate of ALL pediatric patients has been up to $70-80 \%$ (17), and the long-term survival rate has reached approximately $80 \%$ (18), but approximately $20 \%$ cases still suffer from relapse. Only $30-40 \%$ pediatric patients with ALL relapse are cured $(19,20)$ and its mortality rate is high, so it is known as one of the four major tumors in children (the other three are acute leukemia, lymphoma and brain tumor) (21). Therefore, the key to improving the long-term survival of ALL pediatric patients is to reduce its relapse rate, master the major risk factors for ALL relapse and give appropriate treatment.

Intramedullary relapse is the most common in ALL pediatric patients, and the curative effect on it is poor compared with that on extramedullary relapse and intra-extramedullary relapse, and the prognosis is positively correlated with relapse time. Only $10 \%$ pediatric patients with early relapse and $50 \%$ pediatric patients with late relapse can be cured. In this study, there were a total of 71 cases of B-ALL intramedullary relapse. The 3-year EFS rates in early, medium and late stage were $12.5 \pm 7.8 \%, 33.1 \pm 9.8 \%$ and $63.6 \pm 6.1 \%$, respectively. The bilateral log-rank test showed that the prognosis of early relapse was worse than that of late relapse, and the difference was statistically significant $(\mathrm{P}<0.001)$. The MRD level is an independent factor of predicting relapse in chemotherapy (22), which is closely related to the long-term EFS of pediatric patients. Moreover, monitoring the MRD level has an important value in the evaluation of curative effect and adjustment of chemotherapy intensity of leukemia.

ALL relapse was more common in children in IR group and HR group, but the clinical observation found that some SI pediatric patients also suffered from relapse, mostly the medium and late relapse. Therefore, the current risk stratification cannot 
accurately predict the prognosis yet, and the key to reducing ALL relapse in the future is to find new poor prognosis-related genes used to assess the prognosis and guide the treatment.

\section{Acknowledgements}

Not applicable.

\section{Funding}

No funding was received.

\section{Availability of data and materials}

All data generated or analyzed during this study are included in this published article.

\section{Authors' contributions}

$\mathrm{XZ}$ and HW designed the present study and prepared the manuscript; HF, XZ and BS collected all data; HF and BS performed the flow cytometry; GZ, HW and LD analyzed the data; XZ and HW interpreted the data. All authors read and approved the final manuscript.

\section{Ethics approval and consent to participate}

The present study was approved by the Ethics Committee of Jining First People's Hospital (Jining, China). Written, informed consent was obtained from all patients or their parents prior to their inclusion within the study.

\section{Patient consent for publication}

Not applicable.

\section{Competing interests}

The authors declare that they have no competing interests.

\section{References}

1. Hunger SP and Mullighan CG: Acute lymphoblastic leukemia in children. N Engl J Med 373: 1541-1552, 2015.

2. Roy A, Cargill A, Love S, Moorman AV, Stoneham S, Lim A, Darbyshire PJ, Lancaster D, Hann I, Eden T, et al: Outcome after first relapse in childhood acute lymphoblastic leukaemia - lessons from the United Kingdom R2 trial. Br J Haematol 130: 67-75, 2005.

3. Locatelli F, Schrappe M, Bernardo ME and Rutella S: How I treat relapsed childhood acute lymphoblastic leukemia. Blood 120 2807-2816, 2012

4. Raetz EA, Borowitz MJ, Devidas M, Linda SB, Hunger SP, Winick NJ, Camitta BM, Gaynon PS and Carroll WL: Reinduction platform for children with first marrow relapse of acute lymphoblastic Leukemia: A Children's Oncology Group Study. J Clin Oncol 26: 3971-3978, 2008.

5. Nguyen K, Devidas M, Cheng SC, La M, Raetz EA, Carroll WL, Winick NJ, Hunger SP, Gaynon PS and Loh ML; Children's Oncology Group: Factors influencing survival after relapse from acute lymphoblastic leukemia: A Children's Oncology Group study. Leukemia 22: 2142-2150, 2008.

6. Einsiedel HG, von Stackelberg A, Hartmann R, Fengler R, Schrappe M, Janka-Schaub G, Mann G, Hählen K, Göbel U, Klingebiel T, et al: Long-term outcome in children with relapsed ALL by risk-stratified salvage therapy: Results of trial acute lymphoblastic leukemia-relapse study of the Berlin-FrankfurtMünster Group 87. J Clin Oncol 23: 7942-7950, 2005.
7. Lawson SE, Harrison G, Richards S, Oakhill A, Stevens R, Eden OB and Darbyshire PJ: The UK experience in treating relapsed childhood acute lymphoblastic leukaemia: A report on the medical research council UKALLR1 study. Br J Haematol 108: 531-543, 2000.

8. Bailey LC, Lange BJ, Rheingold SR and Bunin NJ: Bone-marrow relapse in paediatric acute lymphoblastic leukaemia. Lancet Oncol 9: 873-883, 2008.

9. Hogan LE, Meyer JA, Yang J, Wang J, Wong N, Yang W, Condos G, Hunger SP, Raetz E, Saffery R, et al: Integrated genomic analysis of relapsed childhood acute lymphoblastic leukemia reveals therapeutic strategies. Blood 118: 5218-5226, 2011.

10. Pieters R, den Boer ML, Durian M, Janka G, Schmiegelow K, Kaspers GJ, van Wering ER and Veerman AJ: Relation between age, immunophenotype and in vitro drug resistance in 395 children with acute lymphoblastic leukemia - implications for treatment of infants. Leukemia 12: 1344-1348, 1998.

11. Borowitz MJ, Devidas M, Hunger SP, Bowman WP, Carroll AJ, Carroll WL, Linda S, Martin PL, Pullen DJ, Viswanatha D, et al; Children's Oncology Group: Clinical significance of minimal residual disease in childhood acute lymphoblastic leukemia and its relationship to other prognostic factors: A Children's Oncology Group study. Blood 111: 5477-5485, 2008.

12. Zhang R, Yang JY, Sun HQ, Jia H, Liao J, Shi YJ and Li G: Comparison of minimal residual disease (MRD) monitoring by WT1 quantification between childhood acute myeloid leukemia and acute lymphoblastic leukemia. Eur Rev Med Pharmacol Sci 19: 2679-2688, 2015.

13. Möricke A, Reiter A, Zimmermann M, Gadner H, Stanulla M, Dördelmann M, Löning L, Beier R, Ludwig WD, Ratei R, et al; German-Austrian-Swiss ALL-BFM Study Group: Risk-adjusted therapy of acute lymphoblastic leukemia can decrease treatment burden and improve survival: Treatment results of 2169 unselected pediatric and adolescent patients enrolled in the trial ALL-BFM 95. Blood 111: 4477-4489, 2008.

14. No authors listed: An International System for Human Cytogenetic Nomenclature (1985) ISCN 1985. Report of the Standing Committee on Human Cytogenetic Nomenclature. Birth Defects Orig Artic Ser 21: 1-117, 1985.

15. Parker C, Waters R,Leighton C, Hancock J,Sutton R, Moorman AV, Ancliff P, Morgan M, Masurekar A, Goulden N, et al: Effect of mitoxantrone on outcome of children with first relapse of acute lymphoblastic leukaemia (ALL R3): An open-label randomised trial. Lancet 376: 2009-2017, 2010.

16. Gao YJ, Zhu XH, Yang Y, Wu Y, Lu FJ, Zhai XW and Wang HS: Prevalence of ETV6-RUNX1 fusion gene in children with acute lymphoblastic leukemia in China. Cancer Genet Cytogenet 178: 57-60, 2007.

17. Mariottini $P$ and Amaldi F: The 5' untranslated region of mRNA for ribosomal protein $\mathrm{S} 19$ is involved in its translational regulation during Xenopus development. Mol Cell Biol 10: 816-822, 1990.

18. Pui CH, Carroll WL, Meshinchi S and Arceci RJ: Biology, risk stratification, and therapy of pediatric acute leukemias: An update. J Clin Oncol 29: 551-565, 2011.

19. Gorman MF, Ji L, Ko RH, Barnette P, Bostrom B, Hutchinson R, Raetz E, Seibel NL, Twist CJ,Eckroth E, et al: Outcome for children treated for relapsed or refractory acute myelogenous leukemia (rAML): A Therapeutic Advances in Childhood Leukemia (TACL) Consortium study. Pediatr Blood Cancer 55: 421-429, 2010.

20. van den Berg H, de Groot-Kruseman HA, Damen-Korbijn CM, de Bont ES, Schouten-van Meeteren AY and Hoogerbrugge PM: Outcome after first relapse in children with acute lymphoblastic leukemia: A report based on the Dutch Childhood Oncology Group (DCOG) relapse all 98 protocol. Pediatr Blood Cancer 57: 210-216, 2011.

21. Gaynon PS: Childhood acute lymphoblastic leukaemia and relapse. Br J Haematol 131: 579-587, 2005.

22. von Stackelberg A, Seeger K, Henze G and Eckert C: Clinical significance of minimal residual disease in childhood acute lymphoblastic leukemia after first relapse. Leukemia 18: 1727-1729, 2004.

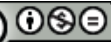

This work is licensed under a Creative Commons Attribution-NonCommercial-NoDerivatives 4.0 International (CC BY-NC-ND 4.0) License. 